\title{
Determinación sistémica de valores tangibles e intangibles y atributos clave para el desarrollo de productos-joya
}

\author{
Medina-Gómez, Andrea Lucía ${ }^{a}$ \& González-Díaz, José Rafael ${ }^{b}$ \\ anniversidad Jorge Tadeo Lozano., Colombia. anmegme@gmail.com. \\ ${ }^{b}$ Pontificia Universidad Javeriana Cali, Colombia. jose.gonzalez@javerianacali.edu.co.
}

\begin{abstract}
Resumen
Análisis de los aspectos clave que influencian la determinación de atributos tangibles e intangibles contenidos en aspectos para el diseño de producto-joya contemporánea, como potenciadores de valores diferenciales de comportamiento y preferencias por parte del consumidor, asi como, por las propias organizaciones del sector en términos del fortalecimiento del capital intelectual, competitividad e identidad, a través del diseño y la gestión del conocimiento.
\end{abstract}

La metodología consideró en primera instancia la recogida de información mediante distintas fuentes documentales (revistas cientificas y profesionales, informes, instituciones, eventos especializados, libros, entre otros). A continuación, se emplearon métodos cualitativos para analizar la opinión de expertos y consumidores en América Latina y Europa, a los efectos de contrastar la importancia de los resultados obtenidos mediante información documental y sesiones grupales. Además, se observan las tendencias en entornos de desarrollo e interacción con el producto a través de distintos canales de comunicación especializados para analizar factores como el diseño, materiales y procesos que contribuyen en la toma de decisiones de compra.

Se destacan los hallazgos sobre componentes relacionales en el producto-joya contemporánea y su contexto en cuanto a la innovación, así como la actuación de atributos clave en la definición del diseño y desarrollo del producto, para el consumidor y los productores del sector. Desde la comprensión de valores tangibles e intangibles, se esboza una caracterización del producto, en el marco de un modelo sistémico de gestión del conocimiento, en el que se presentan evidencias relevantes que pretenden favorecer la toma de decisiones del proceso inicial de diseño de joyería contemporánea, en un campo donde la literatura es limitada. La muestra de expertos es consistente y fiable, se logró consolidar datos en siete países, sin embargo, se espera continuar explorando más contextos geográficos y culturales. Se considera que la diversidad en el origen y procedencia actúan como grupos de control multidisciplinar. El estudio busca reconocer los insights del usuario para contribuir con su satisfacción individual y colectiva, mediante una configuración oportuna del producto.

El estudio posee gran valor especialmente para pequeños fabricantes y diseñadores de joyería como por ejemplo de autor, que buscan mayor participación en el mercado. Los aportes realizados se centran en el ámbito empirico, técnico, académico y empresarial relacionado con el producto-joya contemporánea, así como las aportaciones de relevancia 
detectadas para el investigador y el diseñador; también pueden servir como base para otros estudios enfocados en el diseño y la gestión en sectores productivos basados en los oficios artesanales.

Palabras clave: knowledge, design attributes, intangible values, jewellery industry, identity.

\begin{abstract}
Analysis of the key aspects that influence the determination of tangible and intangible attributes, which are contained in aspects of product design jewel contemporary, as enhancers differential values of behavior and preferences by consumers, as well as by the organizations themselves the sector in terms of strengthening the intellectual, competitiveness and identity capital through design and knowledge management.

In the first instance, the methodology considered the collection of information through distinct documentary sources (scientific and professional journals, reports, institutions, specialized events, books, etc.). Then qualitative methods were used for the analysis the opinion of experts and consumers in Latin America and Europe, in order to contrast the importance of the results obtained through documentary information and group sessions. In addition, trends are observed in development environments and interaction with the product through different communication channels specialised for the analysis factors such as design, materials and processes that contribute in making purchasing decisions.
\end{abstract}

The outstanding findings on relational components in the product-jewel contemporary context in terms of innovation and performance of key attributes in the definition of design and product development for the consumer and producers in the sector. From the understanding of tangible and intangible values, product characterization was outlined as part of a systemic model of knowledge management, in which relevant evidence intended to promote decision making initial process design contemporary jewelry are presented in a field where literature is limited. The expert panel is consistent and reliable. Data were consolidated into seven countries, however, it is expected to continue to explore more geographical and cultural contexts. It is considered that diversity in the origin and source of multidisciplinary groups act as control. The study seeks to recognise the user insights to contribute to their individual and collective satisfaction, through timely product configuration.

The study has great value especially for small manufacturers and jewelry designers such as Signature jewellery, who seek greater market share. Contributions to focus on empirical, technical, academic and business related to the product scope contemporary jewel. The most important contributions are focused on the researcher and designer; they can also serve as a basis for other studies focused on the design and management in productive sectors based on the crafts.

Keywords: a short list with a maximum of five keywords will be included. For example: design, systems, methods, processes, etc. They will be separated by commas.

\title{
1. Introducción
}


En el presente trabajo se ha considerado un conjunto de factores como recursos estratégicos que pueden influir significativamente en la variación y los desarrollos del producto joya; mediante la consideración de contenidos sociales y culturales: el conocimiento como recurso metodológico, tecnológico e inmaterial y la carga simbólica del objeto, ambos, componentes potenciales de innovación que influencian los resultados del diseño y son determinantes en la diferenciación de la joya.

El objetivo principal propuesto en este artículo, se orienta a la valoración de los aspectos clave de diseño como fuente de intangibles (conocimiento, expectativas y deseos, reflejados en los atributos) en la innovación del producto joya contemporánea y su contexto organizacional. El análisis metodológico desde una perspectiva cualitativa y cuantitativa, implicó observar los resultados obtenidos tanto desde la visión del diseño como de los valores propios y culturales del objeto. Se desarrolló un cuestionario dirigido al experto, el cual consideró la intervención de diversas áreas del conocimiento como recurso aportador en la creación de valor del producto, de la innovación y del diseño.

Mediante la información basada en la revisión documental y el análisis de los resultados obtenidos: la opinión del experto en joyería contemporánea, del diseñador con conocimiento específico (modelo sistémico de diseño) y de las expectativas del consumidor, se detallan los atributos de diseño configuradores del producto joya contemporánea contribuyentes, con la innovación y la creación de nuevos productos.

Por otra parte, en la clasificación de los atributos finales; se consideran criterios específicos definidos en forma, función y ergonomía (Hernandis \& Iribarren, 2000) para la determinación de objetivos específicos y portadores clave de información en la fase creativa para el desarrollo del producto-joya contemporánea. En los siguientes epígrafes, se presentan los componentes que aproximan los aspectos considerados en este estudio y su valoración específica.

\subsection{Componentes intangibles y capital intelectual en el desarrollo del producto joya}

\subsubsection{El conocimiento como recurso estratégico y diferenciador: capacidades y habilidades}

Las capacidades y habilidades en el desempeño metodológico y tecnológico se basan tanto en el conocimiento como en la experiencia. Vicente Lorente (2000, p. 114) sostiene que las habilidades individuales idiosincrásicas y la prueba y el error en el proceso conforman el buen hacer del artesano y por lo tanto su experiencia; para esto cita a Barney (1991) en la afirmación referente a que el recurso humano puede constituir ventajas competitivas, siempre y cuando se cumpla con una serie de criterios, para la solución de un problema, por lo que la producción de conocimientos es esencial para mantener el éxito competitivo y organizacional. La capacidad de la organización para renovar y lograr formas novedosas e innovadoras de ventaja competitiva se convierte en lo más importante (Ramezan, 2011, p. 93).

Gil y Bedolla (2009) en búsqueda de nuevas estrategias de aproximación al usuario, para el sector artesano, destacan que contar con las distintas áreas del conocimiento en el trabajo colectivo favorece el emprendimiento para la competitividad, evita la inestabilidad y la pérdida de tradición en el sector. Al igual que el «stock» de recursos tecnológicos, el capital humano puede asociarse a situaciones de especificidad, complementariedad, dependencia histórica y ambigüedad causal que aseguran su inimitabilidad y valor (Becker y Gerhart, 1996 en Vicente Lorente, 2000, 114).

Con relación al diseño, Hernandis e Iribarren (2000: 42-53), plantean el conocimiento como recurso de gestión (a niveles de: explotación, gestión, evolución y mutación) para el desarrollo de productos (en fase teórica, fase constructiva y fase informática), y así, la ejecución del proceso se define a partir de distintos niveles y etapas en la fijación y el logro de los objetivos propuestos. La metodología se sustenta en el 
análisis, la descripción y la detección de problemas al igual que posteriores correcciones en el diseño industrial, ya sean de tipo técnico, de mercado o del conocimiento.

Por otra parte, en el campo cognitivo y emocional sobre productos tecnológicos, se expone que los atributos del objeto están referidos a sus características propias tangibles e intangibles (prestaciones, usos y funciones) que le determinan como objeto multi-atributo y que la combinación de sus atributos, no necesariamente afectan de la misma manera a consumidores y usuarios. Bajo este criterio, sustentan que un producto puede ser percibido desde cualquiera de sus dimensiones, definidas en: la esfera funcional (la novedad tecnológica y la congruencia), simbólica (la similitud visual de los productos existentes), y estética (producto atractivo) Rindova y Petkova, 2007 en Lee, et al. (2011, p. 1196); las cuales pueden ajustarse a características propias del producto joya, debido a su capacidad relacional y comunicativa de los aspectos tecnológicos y simbólicos que representan sus desarrollos y sus formas.

En el marco de los valores que componen el capital intelectual, desde la perspectiva de recursos humanos, know-how y relaciones de la empresa con el entorno, se hace especial referencia al capital relacional que ofrece información acerca de las tendencias o intereses que muestran los agentes de su entorno, los cuales resultan cruciales para detectar oportunidades tecnológicas o de mercado que guíen su proceso de desarrollo de nuevos conocimientos (Martín de Castro, et al., 2009, p. 87). Según Edvinsson \& Sullivan (1996) citados por Hsu \& Fang (2009, p. 668), el capital intelectual mejora el desempeño en el desarrollo de nuevos productos, ya que transforma el conocimiento en valor, pero Hsu \& Fang van más allá al afirmar que los beneficios de nuevos productos provienen más del capital relacional que de la inversión en capital humano o estructural. (2009, p. 673)

No obstante, otro de los recursos intangibles a los que se hace mención, se refieren especialmente al conocimiento acumulado por la empresa (know-how entre otros tipos de conocimientos), donde la fuente principal es el factor humano (Conner, 1991) y se propone el conocimiento (sus dominios y especialidades) como recurso intangible asociado a factores específicos, sociales y culturales (Vecco, 2010) que asegura la creación de valor en los productos, facilita la creatividad, direcciona a la innovación (Pollalis \& Dimitriou, 2008, pp. 310-311) y contribuye con aspectos importantes vinculados al contexto del producto-joya, como por ejemplo, la personalización del objeto mediante la adaptación y experimentación de la técnica y el material (Moraes, 2010).

Además de las consideraciones epistemológicas es importante destacar que estos recursos pueden ser medidos y contabilizados desde la perspectiva de algunos autores como Guthrie, et al. (2012, p. 70) que proponen la contabilidad del capital intangible (ICA por sus siglas en inglés) como una tecnología de gestión, contabilización y rendición de informes hacia el entendimiento, midiendo y reportando los recursos del conocimiento tales como, competencias de los empleados, relaciones con los clientes, marcas, relaciones financieras y las tecnologías de la información y la comunicación, en el desempeño técnico, creativo y de aproximación al consumidor.

\subsubsection{El conocimiento en el marco del producto joya}

Mc Phail expresa que el conocimiento, la característica definitoria de esta nueva época, es ahora considerado como el principal recurso económico, con los nuevos medios y tecnologías de la comunicación, como facilitadores principales de su uso (2009, p. 804). En este contexto, las herramientas tecnológicas se comportan como un medio tangible, que dinamizan las formas del conocimiento (recurso intangible) basadas en la especificidad, la complejidad y la personalización.

En sectores tradicionales donde los procesos idiosincrásicos conforman la identidad y la esencia del producto como sucede con la joya, la inserción tecnológica y sus avances deben proponerse como herramienta-puente a la modernidad sin ser el reemplazo del recurso humano (artesano, diseñador, 
creador de joyas, etc.). Esto implica la conservación de la técnica y de las cargas simbólicas y culturales, características inherentes al objeto, en la definición de los valores intangibles como parte de la significación cultural y de las tendencias actuales de consumo, al mismo tiempo que la adaptación de los procesos demandados por el entorno como lo propone Vecco, (2010); de esta manera, puede garantizarse una vía a la estabilidad del sector joyero en la detección de las nuevas expectativas del usuario (sociales, culturales y emocionales). El valor de las relaciones que mantiene una empresa con los diferentes agentes del entorno con los que se relaciona (principalmente clientes, aliados, proveedores, así como otras empresas e instituciones) o capital relacional, sirven como fuente de información y conocimiento para la propia empresa. (Martín de Castro, et al., 2009, p. 92)

En consecuencia, el conocimiento adquirido como factor que influencia los desarrollos y sus resultados, debe asimilar las oportunidades tecnológicas para la creación de nuevas ideas. Esto apunta también desde una visión organizacional, a la reafirmación del recurso humano como recurso crítico que permite desarrollar y mantener ventajas competitivas basadas en una mayor flexibilidad y capacidad de adaptación al entorno. (Markides y Williamson, 1996 en Vicente Lorente, 2000, 115)

Lo anterior se complementa con la afirmación de Hernandis e Iribarren (2000, p. 53) sobre la gestión del recurso técnico que supone la consideración de características y medios adaptables al alcance del entorno en que se esté trabajando; lo cual necesita contar con el dominio especializado de la técnica y de las herramientas mediadoras para el mejoramiento de la exhibición comunicativa del objeto y el desenvolvimiento creativo. A su vez, los cambios tecnológicos favorecen la exploración técnica y material (experimentación creativa), la aproximación al usuario, la adaptación a ecosistemas actualizados y la creación de ventajas competitivas, que no siempre demanda la pérdida de la tradición, ya que puede ser adaptada a distintos niveles de complejidad necesarios para el sector.

Por lo tanto es necesario destacar que en entornos como el de la joya, debido a su capacidad de conservación, se busca la acentuación de características orientadas a lo único, lo artesanal y lo cultural del objeto como valores intangibles culturales y simbólicos; elementos que resaltan lo propio y lo auténtico (Medina \& Hernandis, (2012, p. 71), como camino para la creación de nuevas ideas. Una empresa con una mejor capacidad para adquirir e integrar conocimiento se desempeñará mejor en el proceso de desarrollo de producto, logrando así un mejor rendimiento en el desarrollo de nuevos productos. (Hsu \& Fang, 2009, p. 673)

\subsection{La experimentación del contexto simbólico de la joya}

Un valor que desempeñan marcas joyeras como por ej. Majorica-España o la plata de Taxco-México (AL Invest IV, 2010) con la marca país, designan a la indicación geográfica un atributo intangible basado en el origen, señal intrínseca relacionada con el producto (Auger, et al., (2010, p. 141) que se imputa a la estética del objeto (Bense en Dorfles, (1972, p. 41); Löbach, (1981, p. 62) y asociada a otros ámbitos vitales que le afirman como condición estratégica de competitividad dentro del sector joyero, donde los componentes materiales son dignos representantes de sus entornos creativos, productivos y de uso.

La experimentación consciente de la técnica y del material como elementos asociados a la carga sígnica del objeto (Barthes, 1993 en Santisteban, 2009), conlleva al desarrollo de características emblemáticas basadas en lo autóctono y lo auténtico. Esto ha permitido que en ecosistemas artesanales como el de la joya (diferentes a los de productos agrícolas o alimenticios, como por ej. vino, café, entre otros) la denominación de origen, también constituya un sistema de características propias del producto (atributos).

De acuerdo con esto, Auger, et al., (2010, p. 145) se refieren a algunos autores en la importancia de la marca considerada como una señal de información importante o atributo intangible (Aaker, 1991; Wernerfelt, 1988), que actúa de manera específica en la caracterización e identificación del usuario a 
través del objeto; y Richardson et al. (1994), describen la marca como un trozo de información que representa un compuesto de la información, reproduce roles dentro del proceso de toma de decisiones de los consumidores, pero la mayor parte de esas funciones giran en torno a una reducción en la incertidumbre.

De acuerdo con lo anterior, Dorfles (1972, p. 69) define el redescubrimiento actual de materiales naturales un hecho participante con la restitución del valor simbólico al objeto gracias a cargas sígnicas, que al igual que las palabras, cobra vida; así las particularidades como el olor o el color, simbolizan pensamientos y creencias (Falchetti, 1999), conforman el sistema comunicativo del objeto (Codina, 2009), y son definidos como incidentes en la personalidad del individuo y del grupo étnico (Korzybski en Dorfles, 1972, p. 85).

En consecuencia, se determinan aspectos clave que regirán los resultados de este estudio: el conocimiento y las adaptaciones tecnológicas, como recursos tangibles e intangibles fundamentales en el desarrollo del producto joya y su contexto relacional, además de atributos que deben estar contenidos en los objetivos de diseño tanto formales, como funcionales y ergonómicos, según el planteamiento sistémico de Hernandis e Iribarren (2000). Asimismo, se plantean las siguientes hipótesis:

H1. El análisis de los componentes relacionales del producto joya y su entorno cultural aporta elementos configuradores clave de innovación y diferenciación para el experto creador de joyas.

H2. Los atributos hacen parte de una o más categorías y pueden actuar bajo dos o más criterios simultáneamente, en términos de forma, función y ergonomía.

H3. A partir de la comprensión de los tangibles e intangibles caracterizadores del producto joya contemporánea, se plantean las cargas predominantes del objeto a partir de valores que se corresponden con categorías estéticas (forma) preminentemente relacionadas con sus cargas simbólicas propias.

\section{Metodología}

La importancia procedimental de este trabajo se enfoca entre otros aspectos, en la presentación de los resultados específicos de la opinión del consumidor y en segunda instancia, del desarrollo metodológico que implicó el tratamiento de las encuestas a expertos en diseño y joyería. Por esta razón, el proceso ha tenido en cuenta la investigación cualitativa y cuantitativa de tipo experimental (Blaxter et. al., (2000); datos e información precedentes, el estudio del consumidor y la información sobre expertos presentados en este artículo, los cuales fueron recogidos principalmente de la revisión documental y de la aplicación de un cuestionario.

La extracción de categorías de los atributos. En esta fase se llevó a cabo un panel compuesto por expertos relacionados con dos campos: el diseño y la joyería, en este último se incluye tanto al artesano joyero como al empresario. Al final del proceso, de acuerdo con las gestiones de análisis realizadas a nivel grupal y perceptual, los atributos más apropiados fueron determinados a partir de categorías sistémicas de diseño definidas en forma, función y ergonomía según el modelo de Hernandis e Iribarren (2000). Los componentes de diseño arriba definidos, son considerados para el análisis del producto joya contemporánea, desde la congruencia de cada uno de estos con el objeto y sus desarrollos.

A partir de la técnica grupal, de la que se realizaron varias sesiones, se determinaron los atributos en tres pasos: en primer lugar, creación de una lista básica de atributos por parte de los expertos (Kleij \& Musters, 2003), en la cual se generaron setenta y tres elementos; en segundo lugar, modificación de ésta 
lista mediante una petición a los participantes para que expresasen sus opiniones acerca de la importancia de los ítems, lo que simplificó el listado. Por último, los expertos generaron un listado final de acuerdo al preliminar y a las opiniones de los participantes (Vigneron \& Johnson, 2004, p. 494); de lo que se obtuvieron treinta atributos, abordados mediante la técnica de diferencial semántico (Osgood et. al., (1957). También se determinan los componentes categóricos de diseño (estética, materia prima, viabilidad técnica, control de calidad, mercado, psicología), basados en la información obtenida a partir de la revisión documental y del contexto. Esto dio lugar a la determinación de los atributos considerados en el cuestionario final.

\subsection{Recogida de datos}

El proceso de aplicación del cuestionario y el contacto con el experto en joyería contemporánea. Este desarrollo incluyó preguntas directamente relacionadas con los atributos específicos y otros factores propios del producto-joya (metodología, tecnología, innovación). El perfil del experto en joyería fue uno de los aspectos importantes a abordar (ver Tabla 1). La construcción del cuestionario, aplicación y recopilación de datos se hizo entre junio de 2011 a enero de 2012; y la puesta en práctica involucró participantes de Latinoamérica y Europa previamente seleccionados (según su experiencia y trayectoria). La asistencia a eventos relacionados con la joya (concretamente en Madrid y Valencia), llamadas telefónicas, el envío del formato online, video conferencias y visitas convenidas en el lugar de trabajo del experto fueron necesarias para la obtención de veinticinco respuestas, con las retroalimentaciones correspondientes. El formato incluyó una guía breve de presentación e introducción, los cuales fueron traducidos (español, inglés y alemán) para una mejor comprensión y la obtención de los datos esperados.

En relación con los atributos configuradores de la joya contemporánea, se planteó un listado inicial de 30 pares opuestos de adjetivos como lo plantearon Vigneron y Johnson, $(2004$, p. 494) lo que permitió obtener, por parte del experto en joyas un total de trece atributos "totalmente importantes". Estos fueron contrastados con resultados previos obtenidos con base en la opinión del consumidor, (catorce atributos) para observar las respuestas coincidentes, que determinaron un listado de diez atributos finales (ver Tabla 2).

La opinión del experto en joyería. A través de la literatura se identificaron tres ítems que afectan los desarrollos de la joya contemporánea definidos en: las metodologías como conceptos estratégicos de inspiración y la tecnología como las nuevas maneras del crear y del hacer, planteados como vectores hacia la innovación (conceptos materiales y herramientas) en la creación de valor del producto; los cuales rigen el cuestionario dirigido a expertos en joyería.

Con respecto a las metodologías, los expertos opinaron que satisfacer la demanda del cliente y proponer una mayor variedad de productos al mercado $(n=9 ; 36 \%)$ son las principales ventajas de su uso para el desarrollo de productos (ver Gráfico 1).

La tecnología como herramienta de apoyo a los desarrollos en el sector joyero (en referencia a tecnologías de información y comunicación, herramientas y maquinaria actualizadas, medios de distribución, etc.), donde las ventajas más representativas se puntualizaron en la redefinición de los medios de promoción y distribución y la aparición constante de materiales nuevos y adaptables a las joyas $(\mathrm{n}=23 ; 92 \%$ respectivamente). La mayor desventaja se determinó la minimización de la exclusividad (ver Gráfico 1). 


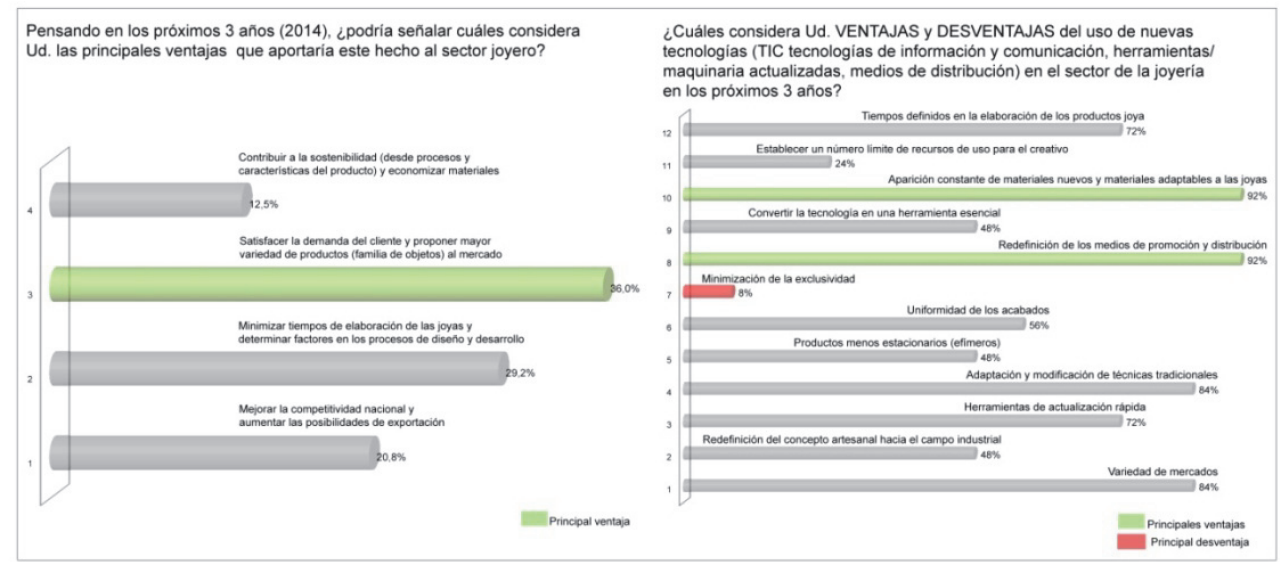

Gráfico 1. Ventajas y desventajas en los desarrollos del experto

Atributos-objetivo de diseño. La definición de atributos que deben estar contenidos en los objetivos del proceso de diseño, constituyen una dimensión cualitativa que se lleva a cabo para evaluar la idoneidad funcional y no funcional de alternativas del producto, frente a diversos criterios tal como lo proponen Sen, et al. (2009, p. 5274). Así, desde la metodología abordada en este estudio, se detallan los atributos finales (ver Tabla 2), basados en la relación atributo-criterios de diseño definidos en forma, función y ergonomía, según el modelo de Hernandis e Iribarren (2000) y son atribuidos a valores porcentuales (ver Gráfico 3) que coadyuvan en la determinación del siguiente paso, en el planteamiento de objetivos que verifiquen los alcances del cumplimiento, la colaboración y la comunicación en el desarrollo de los procesos.

Se exponen conceptos desde la perspectiva del diseño según Hernandis e Iribarren (2000, pp. 60-66), debido a la afinidad entre los términos y relaciones con el producto y el contexto de la joya contemporánea. Los componentes utilizados para este análisis (estética, materia prima, viabilidad técnica, control de calidad, mercado, psicología), son definidos por los autores como variables influyentes en el proceso de diseño y desarrollo de productos; a través de los cuales se determinarán los objetivos formales, funcionales y ergonómicos, como criterios específicos que definen un objeto de diseño. Con base en estos planteamientos, se analizarán a posteriori los objetivos globales pertenecientes a cada uno de los sistemas fundamentales, así como los subobjetivos consecuencia inmediata de la tarea a cumplir por los componentes detectados (Hernandis \& Iribarren, 2000, p. 68).

\section{Resultados}

\subsection{Análisis de los resultados}

El análisis de los datos se hizo mediante Statistical Package for the Social Sciences 11.5 (SPSS). En el listado de atributos el cálculo del coeficiente alpha de cronbach $(\alpha=0,92)$ fue necesario para comprobar la fiabilidad de la escala utilizada (IBM, 2012). Se hallaron frecuencias y medias para confirmar la hipótesis uno (H1), que define el listado final de atributos específicos de diseño, como características propias del objeto y configuradores del producto joya contemporánea.

Perfil del experto en joyería contemporánea. Se analizaron un total de veinticinco cuestionarios aplicados a expertos en joyería con el siguiente perfil: 
Empírico $(\mathrm{n}=4)$, técnico $(\mathrm{n}=7)$, académico $(\mathrm{n}=10)$ y empresa $(\mathrm{n}=4)$, áreas de mayor desempeño en el sector. La ubicación geográfica se consideró según 1) la importancia cultural y artesanal en los objetos de joyería; y 2) el reconocimiento del trabajo contemporáneo y experimental con otros materiales distintos a los más tradicionales. Bajo estos principios se definieron también Latinoamérica $(\mathrm{n}=9 ; 36 \%)$ y Europa (específicamente de España $(\mathrm{n}=9 ; 36 \%)$ y Alemania $(\mathrm{n}=7 ; 28 \%)$ como los lugares de interés. El principio geográfico se tuvo en cuenta, con el fin de implicar diferentes perspectivas en distintos contextos con particularidades idiosincrásicas, como factor influyente en el desarrollo de la joya. En consecuencia, se obtuvo el contacto con expertos en joyas de Argentina, Brasil, Colombia, México, España y Alemania como lugares donde la joyería contemporánea ha hecho presencia de manera importante e innovadora (ICEX, (2012); Eurostat, (2009); LEGISCOMEX, (2007); ESADE, (2011)); el aporte participativo europeo se concentró de la siguiente manera: España con nueve expertos (Valencia, $\mathrm{n}=4 \mathrm{y}$ otras ciudades, como Madrid, Barcelona, Córdoba, Andalucía n=5); Latinoamérica con una muestra igual, y Alemania con siete participantes. Esto permitió un equilibrio entre el panel de expertos.

Tabla 1. Perfil experto en joyería

\begin{tabular}{|c|c|c|c|}
\hline & & $\mathrm{n}$ & $\%$ \\
\hline & Valencia & 4 & 16 \\
\hline & Resto de España & 5 & 20 \\
\hline Ubicación geográfica & Latinoamérica & 9 & 36 \\
\hline & Europa (Alemania) & 7 & 28 \\
\hline & Total & 25 & 100 \\
\hline & 3-5 años & 2 & 8 \\
\hline & 6-12 años & 11 & 44 \\
\hline Fxneriencia & 13-19 años & 3 & 12 \\
\hline Experiencia & 20-30 años & 4 & 16 \\
\hline & $>30$ años & 5 & 20 \\
\hline & Total & 25 & 100 \\
\hline & Especialista & 9 & 36 \\
\hline & Profesional & 14 & 56 \\
\hline & Técnico & 8 & 32 \\
\hline Formación * & Artesano & 15 & 60 \\
\hline & Cursos específicos & 9 & 36 \\
\hline & Otros & 8 & 32 \\
\hline & Total & 25 & 100 \\
\hline & Empírico & 4 & 36 \\
\hline & Técnico & 7 & 56 \\
\hline Área & Académico & 10 & 32 \\
\hline & Empresa & 4 & 60 \\
\hline & Total & 25 & 36 \\
\hline & Tienda & 13 & 54,2 \\
\hline & Taller & 20 & 83,3 \\
\hline & Marketing & 8 & 33,3 \\
\hline Principal actividad * & Investigación & 7 & 29,2 \\
\hline & Distribuidor & 5 & 20,8 \\
\hline & Otros & 4 & 16,7 \\
\hline & Total & 25 & 100 \\
\hline
\end{tabular}

* Aspectos en los que el experto se ubica en más de una categoría 
La experiencia en el tema de las joyas oscila entre 6-19 años (56\%). El nivel formativo se define en dos o más categorías entre especialista, profesional, técnico, artesano y cursos específicos, lo que permitió conocer el tipo de conocimientos entre el recurso humano (ver Tabla 1).

La principal actividad se determinó también en más de una categoría entre tienda, taller, marketing, investigación, distribuidor; lo cual puede deberse a la definición actual y no tradicional sobre la actividad organizacional correspondiente al término empresario (gerentes, consejeros e industriales), bajo el concepto que más que ser personas de negocios independientes se caracterizan por la ejecución del hecho o los hechos que dan sentido a la palabra y la realización de nuevas combinaciones (Schumpeter, 1963, pp. 84-85). La elección del panel consideró para la evaluación, la adecuación de contenidos específicos propios del estudio, en favor de la información requerida a partir de distintas perspectivas en el ámbito de desempeño. Puesto esto en consideración, se valoró formación y experiencia en las áreas correspondientes al campo empírico, académico, técnico y organizacional.

Atributos de diseño configuradores del producto joya contemporánea: la opinión del experto en joyería. Los atributos incluidos en una escala de Likert de siete puntos, donde 1 es nada valorado y 7 totalmente valorado, fueron reducidos a trece como "totalmente importantes" para el experto en joyería, entre los que se obtuvo una media de cinco o superior. Estos fueron definidos en: natural $(\mathrm{m}=5,00)$, diseñado $(\mathrm{m}=5,96)$, distinción del empaque $(\mathrm{m}=5,64)$, moderno $(\mathrm{m}=5,92)$, único $(\mathrm{m}=5,76)$, exclusivo $(5,88)$, artesanal $(\mathrm{m}=5,84)$, alta calidad $(\mathrm{m}=6,04)$, seguro $(\mathrm{m}=5,96)$, especializado $(\mathrm{m}=5,40)$, innovador $(\mathrm{m}=5,80)$, auténtico $(\mathrm{m}=5,92)$ y cómodo $(\mathrm{m}=5,68)$ (ver Tabla 2$)$.

Tabla 2. Atributos de configuración para el desarrollo de una joya contemporánea

\begin{tabular}{l|l|l|l|l|l|l|l|}
\hline \multicolumn{1}{c}{ Media $\alpha=0,92)$} & \multicolumn{3}{c}{$\%$} & \multicolumn{3}{c}{ Media } & \multicolumn{2}{c}{$\%$} \\
\hline elegante (c) & 5,20 & 7 & 28 & emblemático & 4,24 & 3 & 12 \\
\hline natural (e);(c)* & 5,00 & 8 & 32 & artesanal (e);(c)* & 5,84 & 14 & 56 \\
\hline brillante & 4,04 & 4 & 16 & alta calidad (e);(c)* & 6,04 & 15 & 60 \\
\hline permanente (c) & 3,96 & 5 & 20 & complejo & 4,16 & 3 & 12 \\
\hline diseñado (e);(c)* & 5,96 & 16 & 64 & seguro (e);(c)* & 5,96 & 11 & 44 \\
\hline distinción del empaque (e) & 5,64 & 11 & 44 & tecnológico & 5,04 & 4 & 16 \\
\hline moderno (e);(c)* & 5,92 & 12 & 48 & especializado (e) & 5,40 & 8 & 32 \\
\hline único (e);(c)* & 5,76 & 14 & 56 & innovador (e);(c)* & 5,80 & 13 & 52 \\
\hline detallado & 4,08 & 2 & 8 & prestigioso & 5,08 & 6 & 24 \\
\hline sencillo (c) & 5,24 & 131 & 33,2 & costoso & 4,28 & 2 & 8 \\
\hline autóctono & 4,64 & 2 & 12 & de marca & 5,32 & 7 & 28 \\
\hline exclusivo (e) & 5,88 & 13 & 52 & flexible & 5,04 & 5 & 20 \\
\hline auténtico (e);(c)* & 5,92 & 13 & 52 & cómodo (e);(c)* & 5,68 & 8 & 32 \\
\hline sugerente & 5,00 & 6 & 24 & grande & 4,08 & 1 & 4 \\
\hline proporcional & 3,96 & 2 & 8 & ecológico (c) & 5,16 & 7 & 28 \\
\hline
\end{tabular}

(e) atributos totalmente importantes para el experto en joyería; (c) atributos totalmente importantes para el consumidor de joyería. * Atributos concurrentes entre la opinión del consumidor y la opinión del experto en joyería 
Los resultados arriba presentados se contrastaron con las expectativas del consumidor sobre el producto joya contemporánea, determinados en: elegante $(\mathrm{m}=5,01)$, natural $(\mathrm{m}=5,54)$, permanente $(\mathrm{m}=5,29)$, diseñado $(\mathrm{m}=5,70)$, moderno $(\mathrm{m}=5,20)$, único $(\mathrm{m}=5,66)$, auténtico $(\mathrm{m}=5,91)$, artesanal $(\mathrm{m}=5,55)$, alta calidad $(\mathrm{m}=6,18)$, seguro $(\mathrm{m}=6,09)$, innovador $(\mathrm{m}=5,45)$, cómodo $(\mathrm{m}=6,43)$, ecológico $(\mathrm{m}=5,54)$ y sencillo $(\mathrm{m}=5,24)$, también con una media igual a cinco o superior.

Atributos de diseño configuradores del producto joya contemporánea: el contraste de opiniones. El análisis comparativo de los atributos convergentes entre las opiniones del experto-consumidor permitieron obtener resultados más exactos, una reducción de diez atributos: natural, diseñado, moderno, único, auténtico, artesanal, alta calidad, seguro, innovador y cómodo, que especifican las características propias inherentes al producto (ver Gráfico 2). Consolidados estos bajo los componentes sistémicos de diseño: estética, materia prima, viabilidad técnica, control de calidad, mercado y psicología propuestos por Hernandis e Iribarren (2000) como esenciales en el desarrollo de productos.

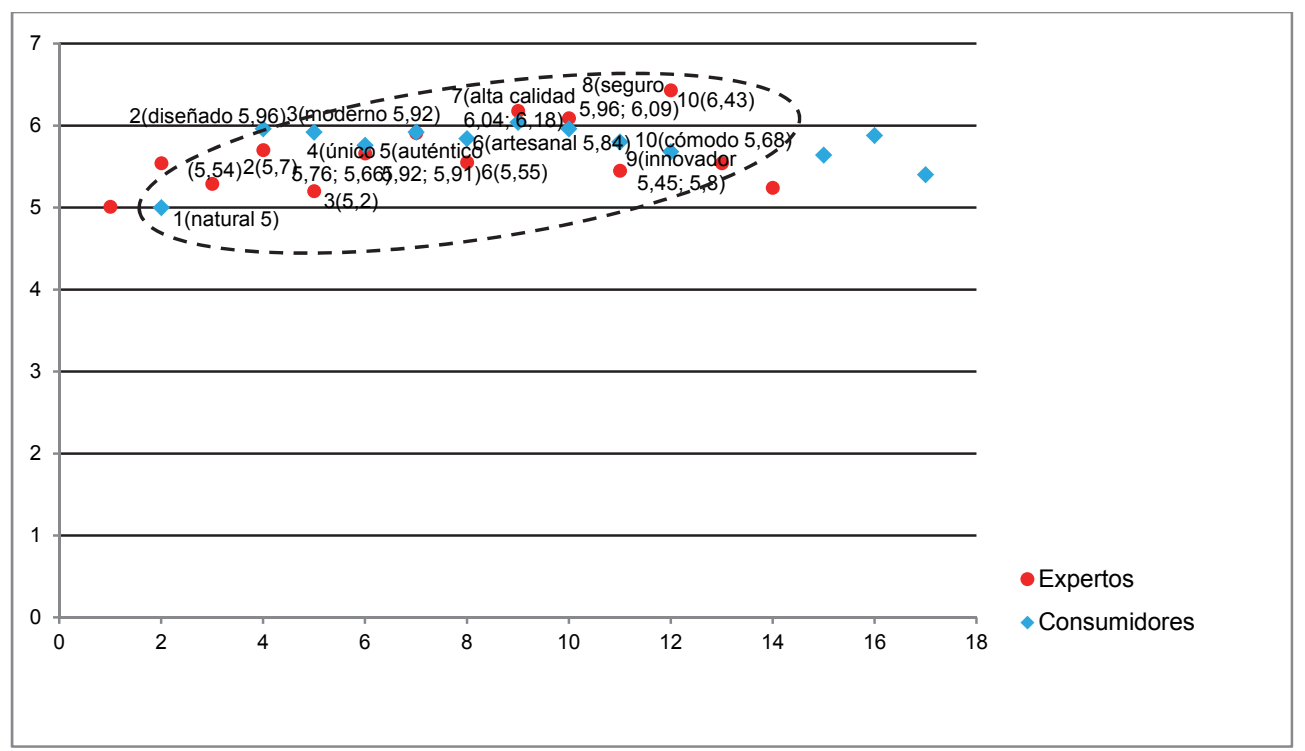

Gráfico 2. Atributos coincidentes entre la opinión del experto en joyería y del consumidor

La definición de los atributos. A partir de los resultados sobre las opiniones confluentes entre expertos (en diseño y joyería) y el consumidor, la revisión documental relacionada con el producto y el diseño (Hernandis e Iribarren, 2000) como participantes en la configuración de la joya contemporánea; el listado final de los atributos más vinculados con en el contexto de la joya, son definidos bajo componentes sistémicos y aspectos asociados al ecosistema de la joya (ver Tabla 3). 
Tabla 3. Relación de atributos con componentes de Diseño

\begin{tabular}{|c|c|c|}
\hline $\begin{array}{c}\text { Atributo de diseño } \\
\text { del producto-joya } \\
\text { contemporánea }\end{array}$ & $\begin{array}{c}\text { Componentes sistémicos } \\
\text { con los que se relaciona } \\
\text { (Hernandis \& Iribarren, } \\
2000)\end{array}$ & $\begin{array}{c}\text { Aspectos sociales, culturales y } \\
\text { tecnológicos asociados con los } \\
\text { desarrollos de la joya }\end{array}$ \\
\hline
\end{tabular}

\begin{tabular}{|l|l|l|l|}
\hline Natural & Materia prims & Valor simbólico, adaptación al entorno \\
\hline Diseñado & & \\
\hline & & & \\
\hline
\end{tabular}

Este análisis de relación presentado en la tabla anterior, demuestra que los atributos son mensurables a través de aspectos metodológicos y tecnológicos, que definen los componentes específicos de diseño (estética, materia prima, viabilidad técnica, control de calidad, mercado, psicología), los cuales son atribuidos a recursos tangibles e intangibles definidos en el conocimiento y la adaptación tecnológica correspondientes a los procesos de elaboración y transformación de un producto determinado y sus contextos de implicación.

Clasificación de los atributos: la opinión del diseñador. La elección del panel de expertos en diseño, requirió especial atención en la consideración de un perfil con conocimientos específicos; de esta manera 
se determinaron los principios de selección en: el conocimiento y la experiencia en diseño y su relación con el modelo sistémico de diseño. Esto con el fin de evaluar objetivamente los contenidos y favorecer la precisión de los resultados desde una visión más especializada.

Para clasificar los diez atributos finales correspondientes al producto joya contemporánea, se pidió a través de un formato online, su clasificación según los criterios de diseño: forma, función y ergonomía (Hernandis \& Iribarren 2000:66); el proceso precisó veintinueve diseñadores docentes y estudiantes de máster en la Universidad Politécnica de Valencia, relacionados con el modelo sistémico de diseño para el desarrollo de productos. En el análisis de los resultados se calculan frecuencias para observar la mayor incidencia en cada uno de los criterios de diseño (forma, función y ergonomía). La clasificación de los atributos finales, según la opinión de los diseñadores fue definida así: diseñado $(55,2 \%)$ y alta calidad (69\%) como aspectos correspondientes a la función; seguro $(62,1 \%)$ y cómodo $(86,2 \%)$ están relacionados con la ergonomía, mientras que natural $(69 \%)$, moderno $(89,7 \%)$, único $(62,1 \%)$, auténtico $(72,4 \%)$, artesanal $(75,9 \%)$ e innovador $(72,4 \%)$ son atribuidos a la forma. También se observó que diseñado fue un atributo común entre las categorías de forma y ergonomía con un valor de 55,2\%, respectivamente (ver Gráfico 3), lo que confirma la hipótesis 2 (H2).

Para la medición de atributos, es necesario destacar, que un atributo puede ser cuantitativo o cualitativo; sin embargo, la técnica de medición desarrollada en esta fase, fue cualitativa. Esta clasificación permitió comprobar cómo son comprendidos y percibidos los atributos inherentes a la joya, desde una perspectiva del diseño; los resultados señalan que los valores predominantes que le caracterizan, pertenecen a criterios relacionados con la forma lo que confirma la última hipótesis propuesta en este artículo (H3). Además, se ha evidenciado la importancia de cada atributo mediante cantidades porcentuales para la configuración del producto joya contemporánea.

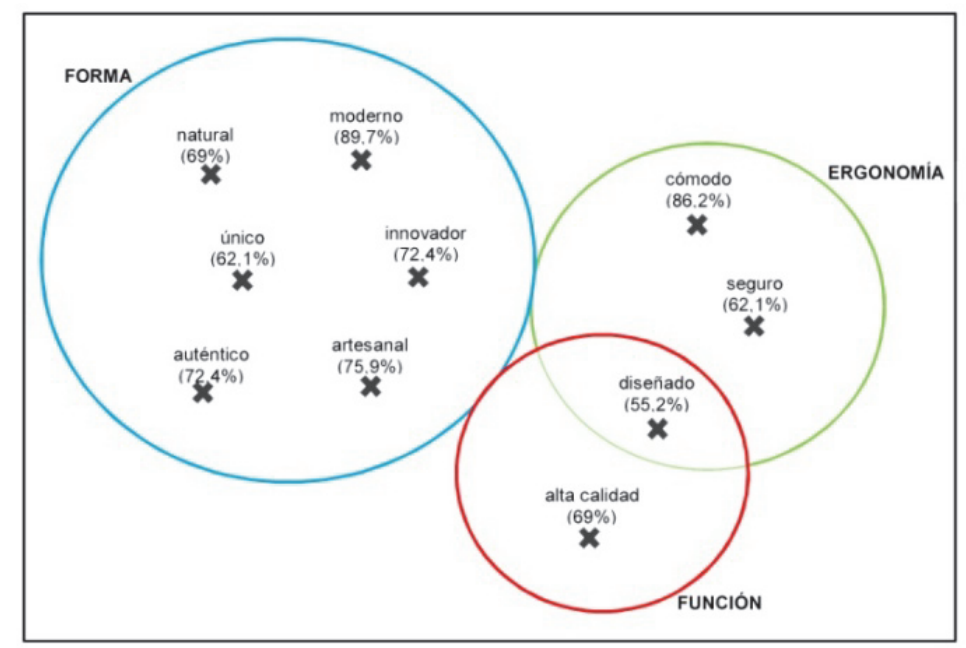

Gráfico 3. Clasificación de atributos según criterios de diseño (forma, función, ergonomía). Ejemplo de la posibilidad de interacción de atributos bajo dos o más criterios simultáneamente. En este caso el atributo diseñado en el ámbito de función y ergonomía.

Finalmente, es necesario destacar que la metodología cualitativa abordada, ha permitido hacer mensurables los atributos. Esta se basó en la clasificación y definición de los mismos, procedimiento fundamental para la indicación de cambios elementales, la toma de decisiones en el proceso de desarrollo y el suministro de información sobre problemas de calidad, comunicación y fallos. Esto permitirá la 
definición de objetivos considerando ciertos atributos clave, como tarea primordial para facilitar la comprensión y el desempeño de su ejercicio.

Otros análisis de relevancia. Teniendo en cuenta la importancia del empleo de métodos óptimos en los procesos de análisis, interpretación y diseño como parte esencial de la concepción y subsecuente fabricación de la joya, la tecnología como recurso actualizado que apoya la creatividad, y ambos, como vectores capaces de impulsar la innovación y la creación de valor del producto; son analizados en este artículo desde las perspectivas planteadas, pero surgen otras premisas que involucran estos dos aspectos, tales como la medición de la variable conocimiento por parte del experto en joyas, en términos de la formación, la profesión, especialidad y experiencia, para reforzar los planteamientos iniciales arriba expuestos. De esta manera, y según este procedimiento se observa lo siguiente:

- Con respecto a las metodologías, los expertos concluyen que las principales ventajas para el sector están enfocadas en satisfacer la demanda del cliente y proponer mayor variedad de productos al mercado ( $\mathrm{n}=9$; $36 \%$ ). Esto pensando a un mediano plazo de 3 años.

- En cuanto a la tecnología, los expertos en joyas estimaron que el porcentaje actual del sector en cuanto al uso de nuevas tecnologías, corresponde a valores entre 25 a 75 por ciento $(25 \%-50 \%$ : $\mathrm{n}=9 ; 36 \%$ y $50 \%-75 \%: \mathrm{n}=7 ; 28 \%$ ); siendo su principal ventaja la redefinición de los medios de promoción y distribución, y la aparición constante de nuevos materiales y adaptables al producto joya $(n=23 ; 92 \%$, respectivamente) y la desventaja más representativa la minimización de la exclusividad ( $\mathrm{n}=23 ; 92 \%)$, seguida de la redefinición del concepto artesanal hacia el ámbito industrial y la estacionalidad de los productos de joyería ( $\mathrm{n}=13 ; 52 \%$, respectivamente).

\section{Conclusiones}

Las conclusiones de este artículo evidencian varias implicaciones prácticas. En primer lugar, los expertos en joyería deben enfocar sus intereses al refuerzo de los recursos intangibles que constituyen el entorno y su trabajo. En segundo lugar, este estudio pretende favorecer la toma de decisiones pertinentes al proceso inicial de diseño de joyas; esto sugiere el tratamiento de la información concreta que puede ser clave en la estrategia de la innovación; y en tercer lugar, se trata de hacer un aporte al experto en joyas, mediante el manejo de información fundamental que posee una fuerte influencia en el criterio de aceptación del producto. Los resultados de análisis cualitativo confirman la relación entre los contextos metodológicos y tecnológicos. Los aspectos y hechos que fortalecen la competitividad del sector se definen en: la experimentación, que permite la creación de otros ámbitos especializados; el dominio del conocimiento como recurso intangible del capital intelectual que propone nuevas lecturas del producto a través de transmitir contenidos emocionales, sociales y culturales; y en la innovación tecnológica como herramienta que contribuye a mejorar el modus vivendi del sector y resalta las características propias de la joya actual basadas en lo natural y lo auténtico. De la misma manera, ha permitido perfilar el listado de atributos, a través de la relación con el diseño, como medio que posibilita la innovación en los desarrollos del producto joya contemporánea.

Por otra parte, los resultados constatan que componentes relacionados con factores culturales, como las cargas sígnicas, afectan al diseño y desarrollo de las características propias del objeto; el cual puede ser interpretado, como elemento de diferenciación de la joya. Asimismo, se obtienen evidencias a favor de la simplificación de los atributos finales como configuradores del producto joya contemporánea; mediante las opiniones obtenidas en la aplicación de los cuestionarios. De esta manera, se confirma la hipótesis 1. No obstante, esta información no es absoluta ya que controlar una característica del proceso no significa necesariamente controlar el proceso; para esto es necesario además, considerar aspectos cuantitativos, que 
sin dejar de ser irrelevantes, no son pertinentes en este abordaje. Por esta razón, es importante comprender que los aportes presentados, se proponen como parte del todo.

En acuerdo con lo anterior, es necesario resaltar que la base de este estudio se orientó a la comprensión, observación y análisis desde una visión cualitativa. Teniendo en cuenta que, gran parte de los datos actuales existentes, se han preocupado por dejar muy bien definido el lado cuantitativo del tema joya, basados por ejemplo en mediciones, pesos, densidades exactas del material y el uso adecuado de las herramientas, también es conveniente incluir aportes que contribuyan al mejoramiento cualitativo del producto y sus procesos.

De esta manera, el estudio se direcciona a la aportación de datos relevantes para la configuración del producto joya contemporánea, mediante el análisis y contraste de los resultados entre las opiniones de expertos y consumidores. La implicación del diseño justifica los valores propios del objeto, a través de conceptos basados en el origen y la tradición como lo demuestran los atributos natural, auténtico y artesanal. Por otra lado, los aspectos considerados previamente como el manejo experimental del material y de la técnica, la especialización y la experiencia en el dominio del conocimiento y de las habilidades se plantean como ventajas estratégicas en la mediación metodológica y comunicativa de lo que se requiere y se espera de la joya (insights) en el entorno actualizado, permitiendo la generación de nuevas ideas.

El análisis cuantitativo permitió observar que los aspectos fundamentales, puntualizados en este artículo en el conocimiento y la tecnología, son comprendidos claramente por el sector joyero, como herramientas que le permitirán establecer una estrecha relación con el usuario directo (92\%) y conocer la valoración de este frente a lo que el creador de joyas le ofrece. De ahí, que el sector joyero debe enfocar sus objetivos creativos y productivos en la actualización y adaptación de sus productos y procesos, mediante la creación de valor en el ecosistema del producto. Por esto, el experto en joyería debe ser consiente del uso adecuado de las herramientas apoyo, que le permitan aproximarse cada vez más al cumplimiento de las expectativas emocionales, sociales y culturales del usuario, en busca de su lealtad.

La hipótesis 2, es verificada a través de los resultados alcanzados en esta investigación, sugieren además que un atributo puede pertenecer o actuar bajo dos o más criterios simultáneamente. Esto se evidencia en la identificación del atributo diseñado, clasificado en forma y ergonomía; lo cual significa que ningún atributo es exclusivo sino que, dependiendo de las condiciones estéticas, comunicativas y del entorno del producto, un atributo puede afectar de forma integrada los resultados del proceso, no siendo incompatible con los demás atributos, sino más bien complementario. Los significados que abarcan los conceptos de conocimiento y tecnología dentro del contexto simbólico del objeto, desde una perspectiva sistémica de diseño (Hernandis \& Iribarren, 2000), fueron percibidos por los diseñadores participantes, dentro de las características propias del producto joya, como signos atribuidos a la estética (forma). Esto teniendo en cuenta la clasificación realizada, a partir de criterios de diseño definidos en forma, función y ergonomía (Hernandis e Iribarren, 2000). De esta manera se valida la última hipótesis (H3) planteada.

Otro aporte clave, se define en la utilidad que supone la medición cualitativa desarrollada. La clasificación de los atributos posibilita una fácil comprensión por parte del experto en joyas sobre la información que sugiere, si esta es requerida para el desarrollo de sus procesos, esta también puede ser considerada de manera total o parcial. Los aportes que provee este estudio, cuentan con la cualidad de ser flexibles y no definitivos, en la mejora de calidad, comunicación y aceptación de producto a diseñar.

Finalmente, el estudio es de gran valor sobre todo para los minoristas de joyería, que buscan el aumento de su cuota de participación en el mercado. El enfoque de los aportes está centrado en la información específica tanto para el empírico, el técnico, académico y el empresario relacionados con el producto joya contemporánea como para el investigador y el diseñador. Además, esta investigación contribuye a la 
incorporación de nuevos datos en el campo de la joya, desde diferentes puntos de vista distintos a los aspectos cuantitativos.

\section{Futuras investigaciones}

Para concluir se pueden señalar interesantes sugerencias de los resultados hacia la investigación futura. En primer lugar, para aproximarse aún más al concepto de percepción del usuario, es importante identificar en tiempo real los insights del consumidor sobre los aspectos tangibles e intangibles que comunica el objeto. Se pretende aplicar otras medidas que superen las insuficiencias de un tratamiento verbo-céntrico que refinen la información y los resultados obtenidos hasta el momento. Para esto se propone el estímulo sensorial con un grupo definido de posibles consumidores de joyas, donde se resalten las características y posibilidades materiales en inmateriales de los atributos propios del producto joya contemporánea. En segundo lugar, con respecto a los resultados obtenidos se propone la incorporación de nuevos aportes teóricos útiles para el estudio desde la perspectiva del diseño y prácticos para el sector de la joyería contemporánea. Por último, un análisis pormenorizado de la actividad de estímulo sensorial debería revelar los aspectos que definen los objetivos de los atributos propuestos, características inherentes al producto. De esta manera, el reto que se plantea enfoca el análisis detallado de las diferentes alternativas metodológicas y de análisis que consideraron este estudio, para así poder extraer conclusiones sobre el efecto que ejercen los tangibles e intangibles metodológicos y tecnológicos como consecuencia de la innovación, así como ofrecer un lenguaje común de los resultados más importantes para el experto relacionado con las joyas.

\section{Referencias}

AL INVEST IV, 2010. La importancia de la moda y la bisutería en el mercado europeo, Bruselas: Dossier especial joyería y bisutería. http://www.al-invest4.eu/attachments/16espanol.pdf

AUGER, P., DEVINNEY, T., LOUVIERE, J. \& BURKE, P., 2010. The importance of social product attributes in consumer purchasing decisions: A multi-country comparative study. International Business Review, 19(2), p. 140159. http://dx.doi.org/10.1016/j.ibusrev.2009.10.002

BLAXTER, L., HUGHES, C. \& TIGHT, M., 2000. Cómo se hace una investigación. Barcelona: Gedisa.

CODINA, C., 2009. Color, texturas y acabados. Barcelona: Parramón.

CONNER, K., 1991. A Historical Comparison of Resource-Based Theory and Five Schools of Thought Within Industrial Organization Economics: Do We Have a New Theory of the Firm?. Journal of management, 17(1), pp. 121-154. http://dx.doi.org/ 10.1177/014920639101700109

DORFLES, G., 1972. Símbolo, comunicación y consumo. Título original: Símbolo, comunicaziones, consumo. Barcelona(Barcelona): Lumen.

ESADE, Universidad Ramón Llull, 2011. Análisis del sector de la joyería y bisutería en los principales mercados europeos. [En línea] Disponible en: http://www.esade.edu/pfw_files/cma/GUIAME/flashes/sectoriales/joyeria.pdf [Último acceso: 1010 2011]. http://www.esade.edu/pfw_files/cma/GUIAME/flashes/sectoriales/joyeria.pdf

EUROSTAT, 2009. European Comission eurostat. [En línea] Available at: http://epp.eurostat.ec.europa.eu/portal/page/portal/statistics/themes [Último acceso: 2912 2009].

FALCHETTI, A., 1999. El poder simbólico de los metales: La tumbaga y las transformaciones metalúrgicas. Boletín de Arqueología, mayo.14(2).

GIL, J. \& BEDOLLA, D., 2009. Redes dinámicas Neo-artesanales. Aplicaciones de la teoría de redes sociales para el estudio del arte popular en México. REDES-Revista hispana para el análisis de redes sociales, 17(10), pp. 210-234. 
GUTHRIE, J., RICCERI, F. \& DUMAY, J., 2012. Reflections and projections: A decade of Intellectual Capital Accounting Research. The British Accounting Review, 44(2), pp. 68-82. http://dx.doi.org/10.1016/j.bar.2012.03.004

HERNANDIS, B. \& IRIBARREN, E., 2000. Capítulo 2. Modelado del Diseño. Editor: Antonio Hervás Jorge, Rafael J. Villanueva Micó ed. Diseño de Nuevos Productos. Una perspectiva sistémica. Valencia: CERES-UPV, pp. 49-97.

HSU, Y. \& FANG, W., 2009. Intellectual capital and new product development performance: The mediating role of organizational learning capability. Technological Forecasting \& Social Change, Junio, 76(5), p. 664-677. http://dx.doi.org/10.1016/j.techfore.2008.03.012

IBM, 2012. IBM. Software. [En línea] Available at: http://www-01.ibm.com/software/analytics/spss/[Último acceso: 2904 2012].

ICEX, Instituto Español de Comercio Exterior, 2012. ICEX-Ranking de los 50 primeros países/territorios destino de la exportación española de MODA. [En línea] Disponible en: http://www.icex.es [Último acceso: 1607 2012].

KLEIJ, F. T. \& MUSTERS, P. D. A., 2003. Text analysis of open-ended survey responses: A complementary method to preference mapping. Journal of Food Quality and Preference, 14(1), pp. 43-52. http://dx.doi.org/10.1016/S09503293(02)00011-3

LEE, S., HA, S. \& WIDDOWS, R., 2011. Consumer responses to high-technology products: Product attributes, cognition, and emotions,. Journal of Business Research, 64(11), p. 1195-1200. http://dx.doi.org/10.1016/j.jbusres.2011.06.022

LEGISCOMEX, 2007. Legiscomex. Joyería y bisutería en Colombia. [En línea] Available at:http://www.legiscomex.com[Último acceso: 1510 2010].

LÖBACH, B., 1981. El proceso de diseño. Edición castellana de Jordi Utgés i Pascual, ed. Diseño Industrial. Bases para la configuración de los productos industriales. edición castellana ed. Barcelona: Gustavo Gili, S.A., pp. 137152.

MARTÍN DE CASTRO, G., ALAMA, E., NAVAS, J. \& LÓPEZ, P., 2009. El papel intelectual en la innovación tecnológica. Una aplicación a las empresas de servicios profesionales de España. Cuadernos de Economía y Dirección de la Empresa, Septiembre, 12(40), pp. 83-109.

MC PHAIL, K., 2009. Where is the ethical knowledge in the knowledge economy?: Power and potential in the emergence of ethical knowledge as a component of intellectual capital. Critical Perspectives on Accounting, 2 Octubre , 20(7), pp. 804-822. doi:10.1016/j.cpa.2008.09.004

MEDINA, A. \& HERNANDIS, B., 2012. La relación artesanía-diseño a través del producto joya. Una perspectiva $\begin{array}{lllll}\text { histórico-técnica. } & \text { Revista } & \text { Iconofacto, } & 8(10), & \text { pp. }\end{array}$ http://revistas.upb.edu.co/index.php/iconofacto/article/view/1356

MORAES, V., 2010. O reaproveitamento de ideias e materiais no design de joias: origem, intertextualidade e sustentabilidade. DAMT: Design, Arte, Moda e Tecnologia, Issue 26, pp. 104-116.

OSGOOD, C. E., SUCI, G. J. \& TANNENBAUM, P., 1957. The Measurement of Meaning. Free Press ed. Urbana, IL: University of Illinois.

POLLALIS, Y. A. \& DIMITRIOU, N. K., 2008. Knowledge mangement in virtual enterprises: A systemic multimethodology towards the strategic use of information. International Journal of Information Management N.28, August, 28(Issue 4), pp. 305-321. http://dx.doi.org/10.1016/j.ijinfomgt.2008.02.005

RAMEZAN, M., 2011. Intellectual capital and organizational organic structure in knowledge society: How are these concepts related?. International Journal of Information Management, Febrero, 31(1), pp. 88-95. http://dx.doi.org/10.1016/j.ijinfomgt.2010.10.004

SANTISTEBAN, Y., 2009. La joya como comunicadora social. Actas de Diseño, Volumen V, p. 52. http://fido.palermo.edu/servicios_dyc/publicacionesdc/vista/detalle_articulo. php?id_libro=88\&id_articulo=2782

SCHUMPETER, J., 1963. Capítulo II: El fenómeno fundamental del desenvolvimiento económico. En: Teoria del desenvolvimiento económico : una investigación sobre ganancias, capital, crédito, interés y ciclo económico. Tercera edición en español ed. México: Fondo de Cultura Económica, pp. 68-103. 
SEN, C., BARAÇLI, H., SEN, S. \& BASLIGIL, H., 2009. An integrated decision support system dealing with qualitative and quantitative objectives for enterprise software selection. Expert Systems with Applications, 36. Parte 1(3), p. 5272-5283. http://dx.doi.org/10.1016/j.eswa.2008.06.070

VECCO, M., 2010. A definition of cultural heritage: From the tangible to the intangible. Journal of Cultural Heritage, Julio-Septiembre, 11(3), p. 321-324. http://dx.doi.org/10.1016/j.culher.2010.01.006

VICENTE LORENTE, J., 2000. Inversión en intangibles y creación de valor en la industria manufacturera española. Economía Industrial, II(332), pp. 109-123. http://dialnet.unirioja.es/servlet/articulo?codigo=297806

VIGNERON, F. \& JOHNSON, L., 2004. Measuring perceptions of brand luxury. Journal of Brand Management, 11(6), p. 484-506. doi:10.1057/palgrave.bm.2540194 